\title{
Comparison between Prediabetes Defined by Hemoglobin A1c (A1C) 5.7- $6.4 \%$ and that Defined by Impaired Fasting Glucose (IFG) in a Japanese Population
}

\author{
Hiroshi Suzuki ${ }^{1}, E \mathrm{jji} \mathrm{Oda}^{2 *}$ and Yoshifusa Aizawa ${ }^{3}$
}

${ }^{1}$ Department of Internal Medicine, Tachikawa Medical Center, Japan

${ }^{2}$ Medical Check-up Center, Tachikawa Medical Center, Japan

${ }^{3}$ Department of Research and Development, Tachikawa Medical Center, Japan

\begin{abstract}
Objective: To investigate prevalence and cardiovascular risk profiles of prediabetes defined by hemoglobin A1c (A1C) $5.7-6.4 \%$ and that defined by impaired fasting glucose (IFG) in Japanese.

Subjects and Methods: We calculated the prevalence of isolated A1C 5.7-6.4\%, isolated IFG, and overlap of A1C 5.7-6.4\% and IFG and compared cardiovascular risk profiles of each group in non-diabetic Japanese 2,274 men and 1,417 women

Results: The prevalence of isolated A1C $5.7-6.4 \%$, isolated IFG, and their overlapping was $6.1 \%, 11.9 \%$, and $9.5 \%$, respectively in men and $13.3 \%, 4.0 \%$, and $6.4 \%$, respectively in women. $\mathrm{HDL}$ cholesterol $(p=0.004$ in men and 0.03 in women), systolic blood pressure ( $p=0.047$ in men and 0.0005 in women), and total bilirubin ( $p=0.0001$ in men and 0.013 in women) were significantly lower in subjects with isolated A1C 5.7-6.4\% compared with those with isolated IFG.

Conclusions: Overlap of A1C 5.7-6.4\% and IFG was not uncommon in Japanese. There were gender differences in the prevalence of isolated A1C 5.7-6.4\% and isolated IFG. A1C 5.7-6.4\% was associated with lower HDL cholesterol and lower bilirubin.
\end{abstract}

Keywords: Prediabetes; Hemoglobin A1c; Impaired fasting glucose; HDL cholesterol; Total bilirubin

\section{Introduction}

Hemoglobin $\mathrm{A} 1 \mathrm{c}(\mathrm{A} 1 \mathrm{C})$ at a range of $5.7-6.4 \%$ was proposed as a marker of prediabetes [1] in addition to impaired fasting glucose (IFG) and impaired glucose tolerance (IGT) although the World Health Organisation has not recommended using A1C for diagnosis of prediabetes. A1C was significantly associated with risks of cardiovascular disease (CVD) and death from any cause adjusting for fasting glucose (FG), while FG was not significantly associated with risks of CVD and death from any cause adjusting for A1C in non-diabetic adults [2]. Saukkonen et al reported that overlap between A1C 5.7-6.4\%, IFG, and IGT was uncommon and isolated A1C 5.7-6.4\% was associated with higher BMI, higher triglycerides, and lower HDL cholesterol compared with isolated IFG among an aging white population [3]. The overlap between A1C 5.7-6.4\% and IFG was only $2 \%$ in their population [3]. In the present study, we investigated prevalence and cardiovascular risk profiles of prediabetes defined by A1C 5.7-6.4\% and that defined by IFG in a Japanese population.

\section{Subjects and Methods}

\section{Subjects}

Between April 2008 and March 2009, 2,444 men and 1,442 women visited our medical check-up center for health screening and gave informed consent. Excluding diabetic subjects from them, 2,274 men and 1,417 women were the subjects in this study. Diabetes was diagnosed as $\mathrm{FG} \geq 7.0 \mathrm{mmol} / \mathrm{L}, \mathrm{A} 1 \mathrm{C} \geq 6.5 \%$ or antidiabetic medication. The protocol for the study was approved by the ethics committee at Tachikawa Medical Center.

\section{Measurements}

After an overnight fast, blood samples were obtained to measure blood levels of A1C, FG, triglycerides, HDL cholesterol, LDL cholesterol, high-sensitivity C-reactive protein (CRP), and total bilirubin. High-sensitivity CRP was measured with nephelometry using N-latex CRP-2 (Siemens Healthcare Japan, Tokyo, Japan). A1C was measured with latex aggregation immunoassay using Determiner HbA1c (Kyowa Medex, Tokyo, Japan). This A1C measuring technique aligned with current recommendations for diagnosis of diabetes by the Japanese Diabetes Society (JDS) and measured A1C values are presented in JDS\%. The intra- and inter-assay coefficients of variation were $0.79 \%$ and $0.85 \%$. Corresponding values in NGSP $\%$ are obtained by adding 0.4 to values in JDS\%. In this paper, A1C values are shown in NGSP\%. LDL cholesterol was measured with a direct surfactant method using Choletest-LDL (Sekisui Medical Inc., Tokyo, Japan). An average systolic blood pressure (BP) and diastolic BP was calculated from two measurements with the subjects in a sitting position after 5 minutes rest. Waist circumference was measured at the level of the umbilicus in standing position. Body mass index (BMI) was calculated as weight in kilograms divided by the square of height in meters.

*Corresponding author: Eiji Oda, Medical Check-up Center, Tachikawa Medical Center, Nagachou 2-2-16, Nagaoka, Niigata, 940-0053, Japan, Tel: 81 258-36-6221; Fax: 81-258-34-1113; E-mail: ijie@venus.sannet.ne.jp

Received November 14, 2011; Accepted November 18, 2011; Published November 22, 2011

Citation: Suzuki H, Oda E, Aizawa Y (2011) Comparison between Prediabetes Defined by Hemoglobin A1c (A1C) $5.7-6.4 \%$ and that Defined by Impaired Fasting Glucose (IFG) in a Japanese Population. J Diabetes Metab 2:153. doi:10.4172/2155-6156.1000153

Copyright: ( 2011 Suzuki $\mathrm{H}$, et al. This is an open-access article distributed unde the terms of the Creative Commons Attribution License, which permits unrestricted use, distribution, and reproduction in any medium, provided the original author and source are credited. 
Citation: Suzuki H, Oda E, Aizawa Y (2011) Comparison between Prediabetes Defined by Hemoglobin A1c (A1C) 5.7-6.4\% and that Defined by Impaired Fasting Glucose (IFG) in a Japanese Population. J Diabetes Metab 2:153. doi:10.4172/2155-6156.1000153

\section{Statistical analysis}

Prevalence of isolated A1C 5.7-6.4\% and isolated IFG was calculated and compared by chi-squared tests. Means of cardiovascular risk factors were compared by ANOVA among subjects with isolated A1C 5.7-6.4\%, isolated IFG, overlapping A1C 5.7-6.4\% and IFG, and normal FG (NFG) and by subsequent Turkey's tests between subjects with isolated A1C 5.7-6.4\% and isolated IFG. Triglycerides and highsensitivity CRP were compared after log transformation. Statistical analyses were performed with Dr SPSS-2 (IBM Japan Inc., Tokyo, Japan). P values of less than 0.05 were considered as significant.

\section{Results}

The means (SDs) or the medians (lower to upper quartiles) of FG and $\mathrm{A} 1 \mathrm{C}$ were $5.19(0.48) \mathrm{mmol} / \mathrm{L}$ or $5.2(4.9-5.4) \mathrm{mmol} / \mathrm{L}$ and 5.39 $(0.29) \%$ or $5.4(5.2-5.5) \%$, respectively in men and $4.93(0.46) \mathrm{mmol} / \mathrm{L}$ or $4.9(4.6-5.2) \mathrm{mmol} / \mathrm{L}$ and $5.40(0.30) \%$ or $5.4(5.2-5.6) \%$, respectively in women. The mean FG was significantly higher in men than in women $(\mathrm{p}<0.0001)$, but the mean A1C was not significantly different between men and women $(p=0.127)$. Prediabetes data are presented in (Table 1 and Table 2). The prevalence of total prediabetes, isolated A1C 5.7-6.4\%, isolated IFG, and their overlapping was $27.5 \%, 6.1 \%, 11.9 \%$, and $9.5 \%$, respectively in men and $23.7 \%, 13.3 \%, 4.0 \%$, and $6.4 \%$, respectively in women. The prevalence of isolated A1C $5.7-6.4 \%$ was significantly lower in men and higher in women than that of isolated IFG. Thus, if A1C was used as the only diagnostic tool instead of fasting glucose, then the prevalence of prediabetes would decrease in men, but increase in women. However, their overlapping was not uncommon. HDL cholesterol ( $\mathrm{p}=0.004$ in men and 0.03 in women), systolic blood pressure ( $\mathrm{p}=0.047$ in men and 0.0005 in women), and total bilirubin ( $\mathrm{p}=0.0001$ in men and 0.013 in women) were significantly lower in subjects with isolated A1C 5.7-6.4\% compared with those with isolated IFG in both men and women. Diastolic BP was significantly lower in subjects with isolated A1C 5.7-6.4\% compared with those with isolated IFG in women $(\mathrm{p}=0.0002)$. Age, BMI, waist circumference, and triglycerides were not significantly different between subjects with isolated A1C 5.7-6.4\% and isolated IFG in both men and women. HDL cholesterol was not significantly different between isolated A1C 5.76.4\% and overlapping A1C 5.7-6.4\% and IFG both in men ( $\mathrm{p}=0.96)$ and women $(\mathrm{p}=0.95)$. While total bilirubin was not significantly different between the two groups in women $(\mathrm{p}=0.999)$, it was significantly lower in isolated A1C 5.7-6.4\% than in overlapping A1C 5.7-6.4\% and IFG in men $(\mathrm{p}=0.039)$.

\section{Discussion}

In contrast to the results reported by Saukkonen et al in an aging white population [3], overlap of A1C 5.7-6.4\% and IFG was not uncommon and the prevalence of isolated A1C $5.7-6.4 \%$ was

\begin{tabular}{|c|c|c|c|c|c|}
\hline & isolated $\mathrm{A} 1 \mathrm{C} 5.7-6.4 \%$ & isolated IFG & overlapping & NFG & $p^{*}$ \\
\hline A1C (\%) & $5.82(0.13)$ & $5.41(0.17)$ & $5.92(0.21)$ & $5.28(0.21)$ & $<0.0001$ \\
\hline FG (mmol/L) & $5.17(0.24)$ & $5.81(0.28)$ & $5.99(0.34)$ & $4.99(0.31)$ & $<0.0001$ \\
\hline n (\%) & $138(6.1)$ & $271(11.9)$ & $217(9.5)$ & $1,648(72.5)$ & $<0.0001$ \\
\hline age (years) & $54.8(9.8)$ & $53.9(8.5)$ & $56.0(8.4)$ & $50.3(9.6)$ & 0.832 \\
\hline BMI $\left(\mathrm{kg} / \mathrm{m}^{2}\right)$ & $24.1(3.8)$ & $23.9(2.9)$ & $24.6(2.8)$ & $22.8(2.6)$ & 0.884 \\
\hline waist circumference $(\mathrm{cm})$ & $86.8(9.4)$ & $86.7(7.9)$ & $88.1(7.6)$ & $83.0(7.5)$ & 0.998 \\
\hline systolic BP (mmHg) & $124(16.9)$ & $128(18.4)$ & $127(18.0)$ & $120(16.8)$ & 0.047 \\
\hline diastolic BP (mmHg) & $78.6(9.6)$ & $80.8(10.9)$ & $80.3(10.7)$ & $76.5(10.5)$ & 0.183 \\
\hline triglycerides $(\mathrm{mmol} / \mathrm{L})$ & $1.32(0.93-1.92)$ & $1.25(0.90-1.76)$ & $1.45(1.06-2.01)$ & $1.11(0.79-1.56)$ & $0.841^{\#}$ \\
\hline HDL cholesterol (mmol/L) & $1.37(0.33)$ & $1.50(0.36)$ & $1.39(0.34)$ & $1.51(0.37)$ & 0.004 \\
\hline LDL cholesterol (mmol/L) & $3.31(0.78)$ & $3.18(0.79)$ & $3.28(0.78)$ & $3.10(0.75)$ & 0.349 \\
\hline total bilirubin (mmol/L) & $12.2(4.4)$ & $14.9(6.1)$ & $14.0(5.4)$ & $14.9(6.4)$ & 0.0001 \\
\hline high-sensitivity CRP (mg/L) & $0.49(0.27-0.81)$ & $0.35(0.19-0.63)$ & $0.46(0.24-0.80)$ & $0.28(0.15-0.57)$ & $0.136^{\#}$ \\
\hline
\end{tabular}

Data are presented as means (SD) or medians (interquartile range); FG, fasting glucose; IFG, impaired FG; NFG, normal FG; BP, blood pressure; CRP, C-reactive protein; $\mathrm{p}^{*}$, isolated A1C 5.7-6.4 vs. isolated IFG; \# compared after log transformation

Table 1: Cardiovascular risk factors of participants with isolated A1C 5.7-6.4\%, isolated IFG, overlapping A1C 5.7-6.4\% and IFG, and NFG in men.

\begin{tabular}{|c|c|c|c|c|c|}
\hline & isolated $\mathrm{A} 1 \mathrm{C}$ 5.7-6.4\% & isolated IFG & overlapping & NFG & $p^{*}$ \\
\hline A1C (\%) & $5.82(0.14)$ & $5.43(0.14)$ & $5.93(0.20)$ & $5.29(0.22)$ & $<0.0001$ \\
\hline $\mathrm{FG}(\mathrm{mmol} / \mathrm{L})$ & $5.07(0.29)$ & $5.75(0.24)$ & $5.92(0.34)$ & $4.78(0.33)$ & $<0.0001$ \\
\hline n (\%) & $188(13.3)$ & $56(4.0)$ & $90(6.4)$ & $1,083(76.4)$ & $<0.0001$ \\
\hline age (years) & $56.3(8.1)$ & $55.7(9.9)$ & $58.6(8.6)$ & 49.7 (9.5) & 0.981 \\
\hline BMI $\left(\mathrm{kg} / \mathrm{m}^{2}\right)$ & $22.7(3.7)$ & $22.7(3.2)$ & $23.3(4.2)$ & $21.3(2.8)$ & 0.999 \\
\hline waist circumference $(\mathrm{cm})$ & $82.1(9.2)$ & $81.1(8.9)$ & $84.4(10.2)$ & $77.8(8.0)$ & 0.861 \\
\hline systolic BP (mmHg) & $114(17.6)$ & $124(19.9)$ & $123(20.1)$ & $111(16.0)$ & 0.0005 \\
\hline diastolic BP (mmHg) & $70.7(10.6)$ & $77.1(11.2)$ & $75.5(11.7)$ & $69.3(9.9)$ & 0.0002 \\
\hline triglycerides $(\mathrm{mmol} / \mathrm{L})$ & $0.98(0.70-1.30)$ & $0.86(0.67-1.13)$ & $0.97(0.77-1.32)$ & $0.79(0.60-1.05)$ & $0.567^{\#}$ \\
\hline HDL cholesterol (mmol/L) & $1.65(0.33)$ & $1.81(0.51)$ & $1.62(0.37)$ & $1.77(0.37)$ & 0.030 \\
\hline LDL cholesterol (mmol/L) & $3.23(0.68)$ & $3.34(0.88)$ & $3.41(0.68)$ & $3.08(0.77)$ & 0.853 \\
\hline total bilirubin (mmol/L) & $11.2(3.6)$ & $13.3(4.0)$ & $11.3(4.0)$ & $12.6(4.7)$ & 0.013 \\
\hline high-sensitivity CRP (mg/L) & $0.30(0.16-0.61)$ & $0.21(0.12-0.44)$ & $0.39(0.18-0.89)$ & $0.20(0.10-0.41)$ & $0.344^{\#}$ \\
\hline
\end{tabular}

Data are presented as means (SD) or medians (interquartile range); FG, fasting glucose; IFG, impaired FG; NFG, normal FG; BP, blood pressure; CRP, C-reactive protein $\mathrm{p}^{*}$, isolated A1C 5.7-6.4 vs. isolated IFG; \# compared after log transformation

Table 2: Cardiovascular risk factors of participants with isolated A1C 5.7-6.4\%, isolated IFG, overlapping A1C 5.7-6.4\% and IFG, and NFG in women 
Citation: Suzuki H, Oda E, Aizawa Y (2011) Comparison between Prediabetes Defined by Hemoglobin A1c (A1C) 5.7-6.4\% and that Defined by Impaired Fasting Glucose (IFG) in a Japanese Population. J Diabetes Metab 2:153. doi:10.4172/2155-6156.1000153

significantly lower in men and higher in women than that of isolated IFG in our Japanese population. HDL cholesterol, systolic BP, and total bilirubin were significantly lower in subjects with isolated $\mathrm{AlC}$ 5.7-6.4\% than those with isolated IFG. But, BMI, waist circumference, and triglycerides were not significantly different between subjects with isolated A1C 5.7-6.4\% and isolated IFG in our Japanese population. It may be related with a substantially lower prevalence of obesity in Japanese compared with white populations [4]. The reason why total bilirubin was significantly lower in isolated A1C 5.7-6.4\% than in overlapping A1C 5.7-6.4\% and IFG in men ( $\mathrm{p}=0.039$ ) was unknown. It may possibly be a chance finding resulted from multiple comparisons.

Mostafa et al compared the two different cutoff points of A1C for prediabetes in a UK population and concluded that A1C $5.7-6.4 \%$ was better than A1C 6.0-6.4\% in white Europeans, but A1C 6.0-6.4\% is a reasonable option in south Asians [5]. In the present Japanese population, the prevalence of isolated A1C 6.0-6.4\% and its overlapping with IFG were $1.1 \%$ and $3.6 \%$ in men and $2.5 \%$ and $2.7 \%$ in women. From ROC curve analysis, the optimal cutoff point for detecting IFG was $\mathrm{A} 1 \mathrm{C} \geq 5.5 \%$ (sensitivity/specificity $71.9 \% / 71.4 \%$ ) in men and $\mathrm{A} 1 \mathrm{C}$ $\geq 5.6 \%$ (sensitivity/specificity $74.7 \% / 76.1 \%$ ) in women. Thus, A1C $\geq$ $5.7 \%$ may be better than $\mathrm{A} 1 \mathrm{C} \geq 6.0 \%$ as the cutoff point of prediabetes. Mann et al reported that the prevalence of isolated A1C 5.7-6.4\%, isolated IFG, and their overlapping was $4.9 \%, 20.5 \%$, and $7.7 \%$ in a US population and concluded that clinicians and health systems should understand the differences and similarities in using A1C or IFG in diagnosis of prediabetes [6].

$\mathrm{A} 1 \mathrm{C}$ was significantly associated with risks of $\mathrm{CVD}$ and death from any cause adjusting for FG, while FG was not significantly associated with risks of CVD and death from any cause adjusting for $\mathrm{A} 1 \mathrm{C}$ in non-diabetic adults [2]. The additional CVD risks of A1C beyond FG may be partly contributed by adverse lipid profiles. A1C levels appear to increase with age [7] and will be influenced by any condition that changes red cell turnover [8]. Higher prevalence of A1C 5.7-6.4\% in women than men may be partly associated with a relatively anemic state of women compared with men. In case of pre-menopausal women, Koga et al reported that erythrocyte indices are associated with A1C, independently of plasma glucose levels, in pre-menopausal women even when they are not anemic [9]. We reported that total bilirubin was negatively associated with A1C independently of other cardiovascular risk factors in apparently healthy Japanese men and women [10]. Bilirubin is a potent antioxidant in human body [11] and increased serum levels of total bilirubin are reported to be associated with reduced risk for CVD in some epidemiological studies [12-16]. Thus, total bilirubin is suggested to be an independent negative risk factor for CVD.

Heianza et al reported that diagnosis of prediabetes by both A1C 5.7-6.4\% and IFG identified individuals with an increased risk of progression to diabetes and the predictive value for progression to diabetes assessed by isolated A1C 5.7-6.4\% was similar to that assessed by isolated IFG in a general Japanese population and the two tests used together could efficiently target people who are most likely to develop diabetes [17]. We reported that the odds ratios (95\% confidence interval) of incident diabetes for metabolic syndrome (MetS), FG $\geq$ $5.6 \mathrm{mmol} / \mathrm{L}$, and $\mathrm{AlC} \geq 6.0 \%$ were 5.39 (2.72-10.7), 9.52 (5.08-17.9), and 33.5 (13.0-86.4), respectively, the areas under receiver operating characteristic curve ( $95 \%$ confidence interval) of diagnosing incident diabetes for FG, A1C, and MetS were 0.82 (0.76-0.88), 0.89 (0.82-0.95), and $0.63(0.53-0.72)$, respectively, the optimal cutoff points of FG and $\mathrm{A} 1 \mathrm{C}$ were $5.3 \mathrm{mmol} / \mathrm{L}$ and $6.0 \%$ respectively, and the population attributable risk fractions of FG $\geq 5.3 \mathrm{mmol} / \mathrm{L}, \mathrm{A} 1 \mathrm{C} \geq 6.0 \%$, and MetS were $59 \%, 86 \%$, and $27 \%$, respectively among a general Japanese population [18]. Thus, A1C 5.7-6.4\% may be a valuable marker of both future diabetes and CVD.

\section{Limitations}

The present study is a cross-sectional one. It is better to study further and for longer time intervals in order to investigate whether people with A1C 5.7-6.4\% are most likely to develop future diabetes and CVD. We were unable to study IGT because OGTTs were not performed. The subjects were not randomly recruited from a community but took the health screening tests voluntarily. Thus, they might be more healthconscious than a general population and the present results should be evaluated by future studies in a more general population.

\section{Acknowledgements}

We thank all subjects who participated in the study and the staff at our center who assisted with data collection.

\section{References}

1. American Diabetes Association (2010) Diabetes and classification of diabetes mellitus. Diabetes Care 33 Suppl 1: S62-S69.

2. Selvin E, Steffes MW, Zhu H, Matsushita K, Wagenknecht L, et al. (2010) Glycated hemoglobin, diabetes, and cardiovascular risk in nondiabetic adults. N Engl J Med 362: 800-811.

3. Saukkonen T, Cederberg H, Jokelainen J, Laakso M, Härkönen P, et al. (2011) Limited overlap between intermediate hyperglycemia as defined by A1C 5.7$6.4 \%$, impaired fasting glucose, and impaired glucose tolerance. Diabetes Care 34: $2314-2316$

4. Ministry of Health, Labor, and Welfere, Japan (2004) The National Health and Nutrition Survey in Japan, 2004. Daiichi Shuppan, Tokyo, Japan (in Japanese)

5. Mostafa SA, Khunti K, Srinivasan BT, Webb D, Gray LJ, et al. (2010) The potential impact and optimal cut-points of using glycated haemoglobin, $\mathrm{HbA} 1 \mathrm{C}$ to detect people with impaired glucose regulation in aUK multi-ethnic cohort. Diabetes Res Clin Pract 90: 100-108.

6. Mann DM, Carson AP, Shimbo D, Fonseca V, Fox CS, et al. (2010) Impact of $\mathrm{HbA1c}$ screening criterion on the diagnosis of pre-diabetes among US adults. Diabetes Care 33: 2190-2195.

7. Pani LN, Korenda L, Meigs JB, Driver C, Chamany S, et al. (2088) Effects of aging on A1C levels in individuals without diabetes. Diabetes Care 31: 1991 1996.

8. The International Expert Committee (2009) International Expert Committee report on the role of the $\mathrm{A} 1 \mathrm{C}$ assay in the diagnosis of diabetes. Diabetes Care 32: $1327-1334$

9. Koga M, Morita S, Saito H, Mukai M, Kasayama S (2007) Association of erythrocyte indices with glycated haemoglobin in pre-menopausal women. Diabet Med 24: 843-847.

10. Oda E, Kawai R (2010) Bilirubin is negatively associated with hemoglobin A1C independently of other cardiovascular risk factors in apparently healthy Japanese men and women. Circ J 75: 190-195.

11. Stocker R, Yamamoto Y, McDonagh AF, Glazer AN, Ames BN (1987) Bilirubin is an antioxidant of possible physiological importance. Science 235: 10431046

12. Schwertner HA, Jackson WG, Tolan G (1994) Association of low serum concentration of bilirubin with increased risk of coronary artery disease. Clin Chem 40: 18-23.

13. Troughton JA, Woodside JV, Young IS, Arveiler D, Amouyel P, et al. (2007) Bilirubin and coronary heart disease risk in the Prospective Epidemiological Study of Myocardial Infarction (PRIME). Eur J Cardiovasc Prev Rehabil 14 79-84

14. Perlstein TS, Pande RL, Beckman JA, Creager MA (2008) Serum total bilirubin level and prevalent lower-extremity peripheral arterial disease. National Health and Nutrition Examination Survey (NHANES) 1999 to 2004. Arterioscler Thromb Vasc Biol 28: 166-172. 
Citation: Suzuki H, Oda E, Aizawa Y (2011) Comparison between Prediabetes Defined by Hemoglobin A1c (A1C) 5.7-6.4\% and that Defined by Impaired Fasting Glucose (IFG) in a Japanese Population. J Diabetes Metab 2:153. doi:10.4172/2155-6156.1000153

Page 4 of 4

15. Kimm H, Yun JE, Jo J, Jee SH (2009) Low serum bilirubin level as an independent predictor of stroke incidence: A prospective study in Korean men and women. Stroke 40: 3422-3427.

16. Oda E, Kawai R (2011) A possible cross-sectional association of serum total bilirubin with coronary heart disease and stroke in a Japanese health screening population. Heart Vessels.
17. Heianza Y, Hara S, Arase $Y$, Saito K, Fujiwara K, et al. (2011) HbA1c 5.7$6.4 \%$ and impaired fasting plasma glucose for diagnosis of prediabetes and risk of progression to diabetes in Japan (TOPICS 3): a longitudinal cohort study. Lancet 378: 147-155.

18. Kaneko M, Suzuki H, Watababe H, Oda E, Aizawa Y (2011) Metabolic syndrome is a poor predictor of incident diabetes compared with hemoglobin $\mathrm{A} 1 \mathrm{c}(\mathrm{HbA} 1 \mathrm{c})$ in a general Japanese population. J Diabetes Metab S: 2. 